\title{
CONTROL ISSUES OF INSERTION DEVICES
}

\author{
Jenny Chen, C. S. Chen, C. J. Wang, C. H. Kuo, K. T. Hsu \\ Synchrotron Radiation Research Center \\ No. 1 R\&D Road VI, Hsinchu Science-Based Industrial Park, Hsinchu, Taiwan, R. O. C.
}

\section{Abstract}

Insertion devices play crucial roles in new generation light source. Magnetic performance is the most important consideration factor during the design and construction phase. However, control performance is also an important issue from operation point of view. Motion control performance, residual filed compensation scheme and control response time for continue change in gap or phase during energy scan scenario is typical consideration to evaluate control performance of insertion devices. How to eliminate orbit excursion due to gap or phase change is also an important issue. Various methods have been used to address the problems. Detailed of these issues about insertion devices control will discuss in this report.

\section{INTRODUCTION}

Insertion devices (IDs) are souls in modern light source. Stringent requirements should be meet in IDs for new generation light source applications. Beside magnetic performance, control performance is equally important from operation point of view. Good control means that the design is balance on various issues. Motion control performance, residual filed compensation scheme, control response time for continues change gap or phase during energy scan scenario and protection mechanisms is typical consideration to evaluate control performance of insertion devices. Orbit issues are connected to magnetic performance directly. Orbit displacement when ID parameters change is unavoidable, especially in low energy storage ring. How to keep orbit fix is a big issue in the operation of storage ring in user mode.

\section{CONTROL ELEMENTS OF INSERTION DEVICES CONTROL}

Control element of various IDs is shown in Fig. 1. Motion control is the most important part of IDs control. Gap and phase are the control variables. Magnet gap can adjust fine as resolution of encoder in sub- $\mu \mathrm{m}$ level by modern motion control technology. However, structure deformation due to strong magnet force is in the order of several tens $\mu \mathrm{m}$. Gap calibration is needed to compensate the defect that is cause by deformation. Achieve good reproducible is very important in IDs mechanical design. Phase control is necessary in elliptically polarized IDs. Requirements of motion control in phase axis are relax compare with gap, micron control resolution is enough and achieves easily. However, strong magnetic force challenges the design of mechanical supports and driver system.

Hierarchical protection mechanisms are used to protect IDs form over travel, taper and stall. Theses mechanisms are essential to prevent vacuum chamber and driver system from damage. Software limit is setting in firmware or control includes travel limit, stall, ...etc. Hardware protection devices include limit switches, tile sensors, hard limits, emergency stop buttons, hardwired logic, ...etc. More than three level protections in motion control loops is provided in IDs control.

For novel IDs to produce elliptically polarization light, some electromagnets or kickers are used to switch helicity dynamically. Special power supply is needed to provide fast switching in helicity. The power supplies are driven by waveform generator usually. Dynamic correction is needed to eliminate orbit transient cause by the residue filed error, local bump leakage, and eddy current effect of vacuum chamber.

Most demanded user allowed change limited ID parameters, such as gap or phase setting and stopping moves for the end-station. These operations need granted by control room operator.

Response time of desired operation is also crucial from experiments point of view. Synchronize the ID gap/phase operation with monochromator scan is needed by user. Helicity switching for mechanical phase switching undulator is also important in speed. Short switching time will benefit the experiments.

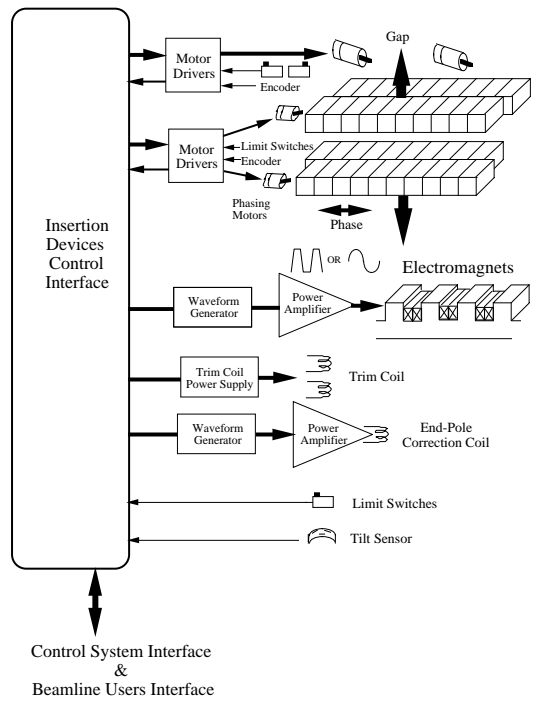

Figure 1: Control element of insertion devices. 


\section{ENGINEERING VIEW TO HANDLE BEAM DYNAMIC ISSUES IN INSERTION DEVICES CONTROL}

There are many beam dynamic issues arise on IDs operation. These issues include dynamic aperture decrease due to reduced symmetry of the lattice by IDs, lifetime reduction cause by operation of Ids, tune changes due to gap change of insertion devices, orbit displacement, ...etc. Insertion devices control related items are summary in following.

Tune changes due to gap change of insertion devices is an issue. The operation of wiggler is fixed parameter operation. Tune shift can lift by appropriate setting of quadrupole by feed-forward techniques. However, tune change of undulator is small $(<0.01)$ compare with wiggler $(\sim 0.05)$. Beam size as well as emittance change due to working point move is small for undulator with proper choice working point.

Imperfection in field integral will cause closed orbit displacement. The main interaction observed between the IDs and the stored electron beam is a small closed orbit displacement. The effect is small in X-ray ring and large in soft X-ray/VUV ring.

Orbit feed-forward is widely used to compensation the orbit displacement dependent on gap change. However, for exotic Ids, gap, and phase are two independent control variables, it is very difficult to use two-dimensional table for the operation of gap and phase at same time. Another possible is use orbit feedback to cope with orbit issues. Global orbit feedback eliminates large perturbation, while local feedback loop to steer photon beam on most stringent beamline.

Most severe effects in orbit control are in various novels IDs, such type IDs include various helical IDs [1][2] include EMW, EEW, APU, EPU, CPU, and some dynamic local bump system [3][4] to extract polarized light. Helicity switching rate in these systems is in $1 \mathrm{~Hz}-$ $100 \mathrm{~Hz}$ order or more. Special dynamic orbit compensation is necessary during operation.

\section{USERS INTERFACE REQUIREMENTS}

Insertion devices users have various requirements for variety experiments. Fixed gap experiment is the easy way for tradition experiments. Most demand user may need change gap and phase during one machine filling cycle or one data scans scenario. For fixed parameters experiment during one machine filled cycle, user may call machine operation set parameter after each filled. For most demand users, they may like set parameter by himself (herself).

For a homogeneous control system that is integrate control in machine and beamlines (e.g. EPICS [5], TACO/TANGO[6]). Users use same control environment as machine control in experiment station, user can integrated control function for their experimental scan program. For heterogeneous control environment (SRRC) user can send their request to control server via local area network.

For electromagnet type devices, there are no movable mechanical parts. The control parameter is excitation current of coils. Excitation may static or dynamic dependent its applications. The user interface is same as mechanical driven wiggler and undulator. Synchronization signal should be provide to synchronized data acquisition system at beamline

Despite types of insertion devices, operators and users can monitor status and initiate actions from the control room or from computers located in offices or on beamlines. Security is maintained by the use of a process variable server that allocates control according to pre-defined access tables. The control system has been designed to enable users to control the ID on their own beamline simply an easily without involving facility operators.

\section{INSERTION DEVICES CONTROL IN TLS}

Taiwan Light Source (TLS) is a $1.5 \mathrm{GeV}$, 3rd generation storage ring. Four straight sections is available to accommodate four insertion devices. Major parameter of IDs is summarized in Table 1.

\begin{tabular}{|l|c|c|c|c|}
\hline \multicolumn{1}{|c|}{ Name } & W20 & U5 & U9 & EPU5.6 \\
\hline Type & hybrid & hybrid & hybrid & pure \\
\hline Length of magnetic period [cm] & 20 & 5 & 9 & 5.6 \\
\hline Number of periods & 13 & 76 & 48 & 66 \\
\hline Minimum magnetic gap [mm] & 22.5 & 18 & 18 & 18 \\
\hline Vertical magnetic field [Tesla] & 1.8 & 0.64 & 1.245 & 0.672 \\
\hline Horizontal magnetic field [Tesla] & - & - & - & 0.453 \\
\hline Horizontal deflection parameter Kx & 33 & 2.99 & 10.46 & 3.52 \\
\hline Vertical deflection parameter Ky & - & - & - & 2.37 \\
\hline Maximum magnetic force [ton] & 20 & 3 & 17 & 3.5 \\
\hline Photon energy [eV] & $800-15000$ & $60-1500$ & $5-100$ & $80-1400$ \\
\hline Total length [m] & 3.0 & 3.9 & 4.5 & 3.9 \\
\hline Installation & Dec. 1994 & Mar. 1997 & Apr. 1999 & May 1999 \\
\hline
\end{tabular}

Table 1: Insertion Devices of TLS (May 1999)

The EPU5.6 is developed in-house [7][8], while the other IDs were contacted to Danfysik and STI Optronics. IDs control in TLS is a heterogeneous system. Local controller of wiggler W20 is a PC/LabVIEW environment and connected to VME crate via IEEE-488 bus. The U5 local control software running in a PC running in MS-DOS, IEEE-488 bus is also used to connect to VME crate via IEEE-488 bus. The U9 and EPU5.6 control are compatible with SRRC control environment. The local controller based upon VME crate equip with PowerPC CPU running LynxOS. The U5 system can execute gap move request within a few seconds for $\mathrm{mm}$ range move. Control system overhead contributes about half of the elapse time for the operation. This is due to the slowly 
operation in local controller through IEEE-488 interface. Overhead of the control system is almost negligible in EPU5.6 and U9 undulators. Current system is work but slightly complicates in maintenance. Cold start procedure is tedious in W20 and U5. Re-engineering of $\mathrm{W} 20$ and U5 control is under planning. The future system will share common hardware and common software as most as possible. Software of EPU5.6 is shown in Fig. 2. All ID control will have similar software structure in near future.

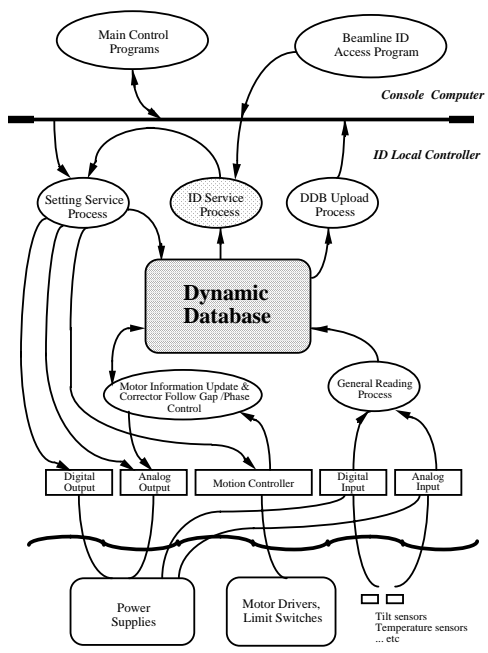

Figure 2: Structure of the EPU5.6 control software.

End-corrector set by a pre-defined value that is function of gap and stored on table are the standard residue filed compensation scheme. The controller has "Fixed" and "Follow" gap option to selected for residue field compensation. All IDs optical encoder is accessible via VME module directly. The update rate is $10 \mathrm{~Hz}$ or $100 \mathrm{~Hz}$ depend on applications.

Beamline users interface have been designed, the user can send command to control server or VME crates directly to perform limited gap/phase setting and stopping. Status of IDs is always available for query in control system. Synchronized ID operation with monochromator in beamline is adequate for the response time point of view.

Beside end-corrector compensation scheme, orbit feed-forward is also used to compensation the orbit excursion. Efforts have been done on U5 and a $1 \mathrm{~m}$ prototype EPU [9]. A few $\mu \mathrm{m}$ in RMS orbit displacement achieve. Eliminate orbit excursion during IDs motion by using orbit feedback that combines global and local is also available in TLS. A few $\mu \mathrm{m}$ RMS orbit displacement control is achievable during IDs motion either gap or phase change. Further improve in BPM performance and corrector control resolution should be done to improve orbit control performance.

EPBM is a dynamic local bump system to extract off-plane polarized light from bending magnet beamline. Functional block diagram of EPBM is shown in Fig. 3. Ideal local bump conditions are difficult to achieve especially in dynamic operation due to local bump ratio error, eddy-current of vacuum chamber, difference response of magnet and power supplies. Dynamic orbit displacement occurs during EPBM operation is also eliminate by dynamic compensation scheme and orbit feedback system. The EPBM are expect work in frequency of $1 \mathrm{~Hz}-100 \mathrm{~Hz}$ either trapezoidal or sine wave excitation soon.

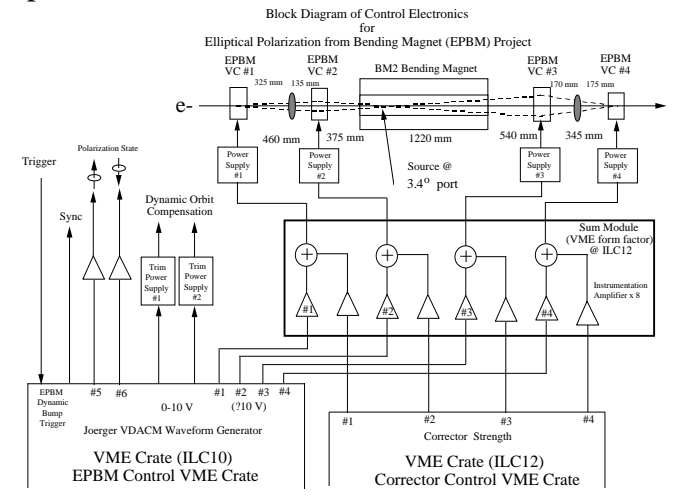

Figure 3: Function block diagram of EBPM dynamic bump system.

\section{SUMMARY}

In this report, discussion in various control issues of insertion devices. Control environment of IDs at TLS is also describe. Good control environment is important to mission critical IDs related experiments. Reliable in control and easily in maintenance is also equally important. Good control and supports are play a key role for the success of experiments that rely on insertion devices..

\section{REFERENCES}

[1] Efim Gluskin, “APS Insertion devices: Recent Developments and Results”, J. Synchrotron Rad. 5 (1988), pp.189-195.

[2] J. Chavanne and P. Elleaume, "Insertion devices at the ESRF", Rev. Sci. Instrum. 66 (1995), p. 1868.

[3] T. Bizen, et al., "Design of a local bump feedback system for a variably polarizing undulator", J. Synchrotron Rad. 5 (1998), pp. $465-467$.

[4] K. K. Lin, et al., " A dynamic local bump system for producing synchrotron radiation with an alternating elliptical polarization", J. Synchrotron Rad. 5 (1998), pp. 398-400.

[5] http://epics.aps.anl.gov/asd/controls/epics/EpicsDocumentation/ WWWPages/EpicsFrames.html.

[6] A. Goetz, et al., "TACO: An Object Oriented Control System for Pcs runing Linux, Windows/NT, OS-9000 or LynxOS", Proc. PCaPAC96, DESY Hamburg, 1996.

[7] K. T. Pan, et al., "Control for Elliptically Polarizing Undulator at SRRC", Proceeding of the ICALEPCS' 97, Beijing, November 37, 1997, p. 614.

[8] K. T. Pan, et al., "The Control performance of Elliptically Polarizing Undulator at SRRC", Proceeding of the 6th European Particle Accelerator Conference EPAC-98, Stockholm, 22-26 June, 1998, p. 2231.

[9] H. P. Chang, et al., "Modelling modulation and dynamic tuning of insertion devices at SRRC", Proceeding of the 5th European Particle Accelerator Conference EPAC-96, Barcelona, 10-14 June 1996, p. 694. 